\title{
Access to primaquine in the last mile: challenges at the service delivery points in pre-elimination era, Myanmar
}

Kay Thwe Han ${ }^{1,2+}$, Khin Thet Wai ${ }^{3^{*}+}$, Tin Oo ${ }^{2}$, Aung Thi ${ }^{4}$, Zayar Han ${ }^{5}$, Daw Kyin Hla Aye ${ }^{5}$, Aung Ye Naung Win ${ }^{6}$ and Jetsumon Sattabongkot ${ }^{1}$

\begin{abstract}
Background: Alongside monitoring of the disease burden, the successful move towards malaria elimination relies on the readiness of the health care delivery system. However, there is a lack of evidence in the gap of existing National Guidelines and access to low dose primaquine in real practice under varying degrees of antimalarial resistance in the pre-elimination phase in Myanmar. Therefore, this study addressed the essential information from the service delivery points (SDPs) of public and private sectors on the availability and the use of primaquine in both supply and demand side. Concomitantly, the study aimed to underscore challenges in health system infrastructure to promote the sustained flow in rolling out primaquine in line with National Guidelines for malaria elimination.
\end{abstract}

Methods: A cross-sectional study conducted from September 2017 to February 2018 included six townships of three states/regions. The team used an observation checklist for documenting primaquine supplies at SDPs. Semi-structured interviews, key informant, and in-depth interviews focused both public and private sectors including staff from the Vector-Borne Diseases Control (VBDC) teams in each state/region and rural health centers $(n=25)$, those from the non-governmental organizations (NGOs), general practitioners and drug sellers $(n=11)$, and recently infected malaria patients $(n=11)$. Triangulation of quantitative and qualitative data provided meaningful interpretations.

Results: Public sector staff reported an adequate stock of primaquine, but it was unavailable at the general practitioners' clinics without any connection to NGOs and also at the unlicensed drug shops. Health care providers of the public sector experienced challenges in poor compliance of malaria patients to primaquine treatment in conjunction with an artemisinin-based combination therapy, loss-to-follow-ups especially in conflict areas, and delays in timely substitution of new batches of primaquine. Respondents from the private sector demanded for the refresher training course on updated antimalarial treatment guidelines.

Conclusion: Monitoring compliance and safety of primaquine treatment was found as a barrier especially among mobile migrant workers and those who were in conflict areas. An alternative strategy by the NMCP could enable to prevent the underutilization of primaquine in vivax malaria to reach the malaria elimination targets.

Keywords: Primaquine, Sustained access, Service delivery points, Antimalarial treatment guidelines, Pre-elimination, Myanmar

\footnotetext{
* Correspondence: khinthetwaidmr@gmail.com

${ }^{\dagger}$ Kay Thwe Han and Khin Thet Wai contributed equally to this work.

${ }^{2}$ DMR, Yangon, Myanmar

Full list of author information is available at the end of the article
} 


\section{Background}

An access to a WHO-recommended low-dose primaquine for gametocytocidal action is critical in global elimination settings as one of the malaria transmission-blocking tools [1]. The National Malaria Control Program (NMCP) in Myanmar has launched the National Malaria Elimination Plan (2016-2030) for accelerating the progress towards zero indigenous cases. Above all, due to the considerable decrease in Plasmodium falciparum malaria, Plasmodium vivax is emerging and dominating in some endemic areas. However, malaria still prevails in hard-to-reach areas with an emerging evidence of drug resistance [2-4]. The National Malaria Treatment Guidelines indicate the use of artemisinin combination therapy (ACT) in conjunction with a gametocytocidal dose of primaquine of $0.25 \mathrm{mg} / \mathrm{kg}$ for both falciparum (single dose) and vivax malaria (8 weeks regimen) [5]. Alongside monitoring of the disease burden, the successful move towards malaria elimination relies on the readiness of the health care delivery system in conformity to other endemic regions [6-8]. However, there is a lack of evidence in the gap of existing National Guidelines and access to low dose primaquine in real practice under varying degrees of antimalarial resistance in the pre-elimination phase. Therefore, this study addressed the essential information from the service delivery points (SDPs) of public and private sectors on the availability and the use of primaquine in both supply and demand side. Concomitantly, the study aimed to underscore challenges in health system infrastructure to promote the sustained flow in rolling out primaquine in line with the National Guidelines for malaria elimination.

\section{Methods}

A cross-sectional study was conducted from September 2017 to February 2018 in six study sites of three states/ regions designated for malaria elimination by 2030 . The study population encompassed the public sector health staff of the NMCP and at the SDPs in rural areas, those from the private sector viz., staff of non-governmental organizations (NGO), general practitioners (GP), and drug sellers, and recently infected malaria patients.

\section{Sampling and sample size}

In each study site, the research team purposively selected two out of six to eight rural health centers (RHCs) with a higher number of reported malaria cases compared to others. At least $80 \%$ of health staff $(n=36)$ who were responsible to diagnose and treat malaria-infected patients, three informants from state/regional VBDC teams, one senior program official from the NGO, and ten respondents (seven GP and three drug sellers) were purposively selected for key-informant interviews (KII) and in-depth interviews (IDI). In addition, the research team used convenience sampling to recruit recently infected malaria patients $(n=11)$.

\section{Data collection methods}

The research team used the observation checklist to record previous access to primaquine, details of drug supply, and the number of malaria cases tested and treated in each SDP at different levels along the supply chain (rural, township, and state/region). Then, trained interviewers conducted the semi-structured interviews of health care providers from the public sector $(n=25)$ as well as from the private sector $(n=11)$ by using the pre-tested and modified questionnaire to uncover their knowledge and experiences in prescribing primaquine, adherence to National Treatment Guidelines and challenges encountered. Pre-tested guidelines for KII and IDIs covered similar issues as semi-structured interviews in the private place. In addition, trained interviewers assessed the use and compliance to primaquine among recently infected malaria patients $(n=12)$, and anonymity and confidentiality issues were strictly observed.

\section{Data management}

Quantitative data entry and analysis were carried out by SPSS version 23.0. After thematic analysis of qualitative data, triangulation of quantitative and qualitative data was done for meaningful interpretations.

\section{Results}

\section{Access to primaquine}

In the public sector, primaquine was available at the respective health facilities without any expired stock according to the clinic registers. In contrast, in the private sector, only five out of eight GPs' clinics and the SDPs of two international NGOs had primaquine. Concerning the supply chain at the public sector, all SDPs had received primaquine from the state/regional VBDC teams. The nature of the supply chain was the "pull system," a need-based system to fill up antimalarials either monthly or quarterly. At these SDPs, they did not report any stock-outs of primaquine within last 3 months. At the private sector, GP clinics supported by private not-for-profit NGOs were fully equipped with primaquine. However, other GP clinics without any NGO support purchased antimalarials from the retail market except for primaquine. The persons responsible for logistics management of two INGOs did not report any primaquine stock-outs in SDPs. Besides, they had a system to regular exchange of nearly expired batches with new batches of primaquine. Primaquine was not available at any of the drug shops surveyed. In some uncomplicated falciparum malaria and confirmed vivax malaria cases, primaquine use was not in line with treatment guidelines among providers in the private sector (Table 1). 
Table 1 Reported antimalarial treatment practices of health care providers in six study sites of three states/regions, Myanmar ( $n=32$ )

\begin{tabular}{|c|c|c|c|c|c|}
\hline Health care & Treatment & Study region $\mathrm{A}$ & Study region $B$ & Study region $\mathrm{C}$ & Total \\
\hline \multicolumn{6}{|c|}{ Confirmed uncomplicated falciparum malaria } \\
\hline \multirow[t]{2}{*}{ Public sector $(n=24)$} & $\mathrm{ACT}+\mathrm{PQ}$ (correct dose and course) & $6 / 6(100 \%)$ & $10 / 10(100 \%)$ & $8 / 8(100 \%)$ & $24 / 24(100 \%)$ \\
\hline & Artesunate 2 tds $\times 3$ days & 0 & 0 & 0 & 0 \\
\hline Private sector $(n=8)$ & $\mathrm{ACT}+\mathrm{PQ}$ (correct dose and course) & $1 / 2(50 \%)$ & $1 / 2(50 \%)$ & $3 / 4(75 \%)$ & $5 / 8(65 \%)$ \\
\hline \multicolumn{6}{|l|}{ Confirmed vivax malaria } \\
\hline \multirow[t]{2}{*}{ Public sector $(n=24)$} & CQ + PQ (correct dose and course) & $8 / 8(100 \%)$ & $5 / 5(100 \%)$ & $11 / 11(100 \%)$ & $24 / 24(100 \%)$ \\
\hline & CQ only & 0 & 0 & 0 & 0 \\
\hline \multirow[t]{2}{*}{ Private sector $(n=8)$} & CQ + PQ (correct dose and course) & $0 / 3$ & $0 / 4$ & $0 / 1$ & $0 / 8$ \\
\hline & CQ only & 0 & 0 & 0 & 0 \\
\hline
\end{tabular}

$A C T$ artemisinin combination treatment, $P Q$ primaquine, $C Q$ chloroquine, $t d s$ three times a day

\section{Challenges in sustained use of primaquine}

The long course of primaquine treatment for 8 weeks in vivax patients was the main reason of poor compliance reported by health staff from the public sector. Mostly, patients rarely returned after 2 weeks. Moreover, health staff felt that it was time-consuming and costly for them to travel from SDPs in rural areas to the Township Health Department for substitution of the nearly expired primaquine with new batches. Respondents further addressed migrant workers as the risk groups in loss-to-follow-ups and found as difficult to monitor their treatment compliance and outcome. They had confronted the similar issue in conflict areas. On the other hand, health care providers of the private sector stated challenges such as a lack of chance to attend the continuing medical education sessions or the refresher training course on updated antimalarial treatment guidelines. Besides, they were more or less not familiar to the ideal treatment of malaria due to the decreasing number of malaria cases and infrequent experience. Primaquine was not available at the retail market, and ACT was the only treatment provided for malaria cases at private clinics without NGO support.

Also, they were not able to monitor the patients' compliance as they failed to turn-up following recovery from the recent malaria episode.

\section{Opinions to improve primaquine treatment}

In the public sector, the respondents preferred the shorter course of primaquine regimen in malaria due to Plasmodium vivax for better compliance than at present. Those from the remote areas would like to opt for the provision of primaquine within the acceptable expiry date to prevent immediate stock-outs and to improve easy access. In the private sector, the availability of primaquine in the retail markets could improve an easy access to GPs so as to treat the patients in accordance with National Treatment Guidelines as required.

\section{Discussion}

In Myanmar, in low malaria endemic sites targeted for elimination by 2030 , vivax malaria reemerged compared to the lower prevalence of falciparum malaria [3]. This study clearly demonstrates the critical role of the updated National Treatment Guidelines to be available for service providers especially in the private sector apart from the sustained access to primaquine in pre-elimination phase. On the other hand, prevailing parasite species in the low endemic settings moving towards elimination influenced the treatment decisions for primaquine and lack of monitoring for compliance to full course in both sectors. In falciparum malaria, the use of SLD primaquine and the adherence to treatment guidelines and compliance were well monitored as reported by key informants in this study similar to African context [9]. In vivax malaria, the longer course and the higher dose of primaquine were needed for radical cure to clear liver stage parasites which was the main reason for non-compliance to full course in this study. Public not-for-profit service providers raised this issue during key informant interviews for their reluctance to use primaquine rather than the safety concerns about hemolysis in Glucose-6-phosphate dehydrogenase (G6PD) deficiency and other contraindications as expected which was consistent to other studies $[10,11]$. If vivax malaria is to be eliminated, the use of radical curative treatment requires attention. Due to divergence in policy and practice, primaquine is underused [12]. To date, Thailand and Cambodia have adopted malaria elimination in their national strategic plans to achieve zero indigenous case status by 2024 and 2025, respectively. Both country programs plan to introduce or revise guidelines for safe, effective radical cure for vivax malaria with a routine G6PD testing [11, 12]. However, policy alone is not sufficient. Programs can only gain from the policy if they are capable for planning and implementation in hard-to-reach areas. Allowing for sufficient time and specific guidance is 
critical to prepare for training and other activities to promote the use of low-dose primaquine particularly in remote sites.

\section{Limitations of the study}

The study period covered only the second peak of malaria transmission season in Myanmar. Very few malaria patients could join the interviews and were unable to capture their constraints in compliance to long course of primaquine in vivax malaria.

\section{Conclusion}

In both public and private sectors, monitoring compliance and safety of primaquine treatment was a barrier especially among mobile migrant workers and those who were in conflict areas. Introducing an alternative strategy by the NMCP is a necessity to improve treatment options and to prevent the underutilization of Primaquine for gametocytocidal effect intended in vivax malaria to reach the malaria elimination targets.

\section{Abbreviations}

ACT: Artemisinin combination therapy; GP: General practitioner; IDI: In-depth interviews; Kll: Key informant interviews; NGO: Non-governmental organizations; NMCP: National Malaria Control Program; RHC: Rural health center; SDPs: Service delivery points; VBDC: Vector-Borne Diseases Control

\section{Acknowledgements}

Authors are grateful to Dr. Kyaw Zin Thant, Director General, Department of Medical Research for his continued support and encouragement. Last but not the least, thanks are due to respondents at different levels of health system for their active participation.

\section{Funding}

Ministry of Health and Sports, Myanmar funded for carrying out the implementation research.

\section{Availability of data and materials}

Data will be available upon reasonable request made to the first author.

\section{Authors' contributions}

$\mathrm{KTH}, \mathrm{AT}, \mathrm{JP}, \mathrm{TO}$, and KTW conceptualized and generated the idea for the study. KHA and ZYH supervised field data collection conducted by PZA and one project staff. Overall data analysis was managed by KTH, TO, AYNW. KTH and KTW drafted the manuscript. All authors read and approved the final manuscript.

\section{Ethics approval and consent to participate}

The Ethics Review Committee, Department of Medical Research approved the study. Privacy, anonymity, and confidentiality issues were ensured following Helsinki Declaration.

\section{Consent for publication}

Not applicable

\section{Competing interests}

The authors declare that they have no competing interests.

\section{Publisher's Note}

Springer Nature remains neutral with regard to jurisdictional claims in published maps and institutional affiliations.

\section{Author details}

${ }^{1}$ Mahidol Vivax Research Unit, Faculty of Tropical Medicine, Mahidol University, Nakhon Pathom, Thailand. ${ }^{2}$ Parasitology Research Division, Department of
Medical Research (DMR), No. 5 Ziwaka Road, Yangon 11191, Myanmar. ${ }^{3}$ DMR, Yangon, Myanmar. ${ }^{4}$ National Malaria Control Program, Department of Public Health (DoPH), Yangon, Myanmar. ${ }^{5}$ Parasitology Research Division, DMR, Yangon, Myanmar. ${ }^{6}$ Epidemiology Research Division, DMR, Yangon, Myanmar.

Received: 14 July 2018 Accepted: 10 September 2018

Published online: 18 September 2018

\section{References}

1. WHO. Strategy for malaria elimination in the Greater Mekong Subregion: 2015-2030. Manila: World Health Organization Regional Office for the Western Pacific; 2015.

2. Global Malaria Programme. WHO Malaria Policy Advisory Committee (MPAC) meeting. Meeting Report, 2017. http://apps.who.int/iris/bitstream/ handle/10665/259396/WHO-HTM-GMP-2017.21-eng.pdf

3. Department of Public Health. National strategic plan for intensifying malaria control and accelerating progress towards malaria elimination (2017-2021). Naypyidaw: MoHS, The Republic of the Union of Myanmar; 2016. www. mohs.gov.mm/

4. NMCP. National plan for malaria elimination in Myanmar (2016-2030). Naypyidaw: MoHS, The Republic of the Union of Myanmar; 2015.

5. WHO and GMP. Single dose primaquine as a gametocytocide in Plasmodium falciparum malaria. Updated WHO policy recommendation (October 2012). http://www.who.int/malaria/pq_updated_policy_ recommendation_en_102012.pdf

6. WHO. WHO evidence review group: The Safety and Effectiveness of Single Dose Primaquine as a P. falciparum gametocytocide. Meeting Report. 2012. http://www.who.int/malaria/mpac/sep2012/primaquine_single_dose_pf_ erg_meeting_report_aug2012.pdf

7. Tun KM, Imwong M, Lwin KM, Win AA, Hlaing TM, Hlaing T, et al. Spread of artemisinin-resistant Plasmodium falciparum in Myanmar: a cross-sectional survey of the K13 molecular marker. Lancet Infect Dis. 2015;15:415-21.

8. Ashley EA, Recht Jand White NJ. Primaquine: the risks and the benefits. A review. Malaria J. 2014;13:418-30.

9. Chen I, Poirot E, Newman M, Kandula D, Shah R, Hwang J, Cohen JM, Gosling R, Rooney L. An assessment of the supply, programmatic use, and regulatory issues of single low-doseprimaquine as a Plasmodium falciparum gametocytocide for sub-Saharan Africa. Malar J. 2015;14:204-23.

10. Graves PM, Choi L, Gelband H, Garner P. Primaquine or other 8aminoquinolines for reducing Plasmodium falciparum transmission. Cochrane Database Syst Rev. 2018;Issue 2. Art. No.:CD008152. https://doi. org/10.1002/14651858.CD008152.pub5.

11. Recht J, Ashley EA, White NJ. Use of primaquine and glucose-6-phosphate dehydrogenase deficiency testing: divergent policies and practices in malaria endemic countries. PLoS Negl Trop Dis. 2018;12(4):e0006230 https:// doi.org/10.1371/journal.pntd.0006230.

12. Kitchakarn S, Lek D, Thol S, Hok C, Saejeng A, Huy R, Chinanonwait $\mathrm{N}$, Thimasarn K, Wongsrichanalai C.Implementation of G6PD testing and primaquine for $P$. vivax radical cure: operational perspectives from Thailand and Cambodia.WHO South-East Asia J Public Health 2017, 6(2): 60-68.
Ready to submit your research? Choose BMC and benefit from:
- fast, convenient online submission
- thorough peer review by experienced researchers in your field
- rapid publication on acceptance
- support for research data, including large and complex data types
- gold Open Access which fosters wider collaboration and increased citations
- maximum visibility for your research: over $100 \mathrm{M}$ website views per year
At BMC, research is always in progress.
Learn more biomedcentral.com/submission 